\title{
ERRATUM
}

\section{Erratum - Effect of Tramiprosate in Patients with Mild-to-moderate Alzheimer's disease: Exploratory Analyses of the MRI sub-group of the Alphase study}

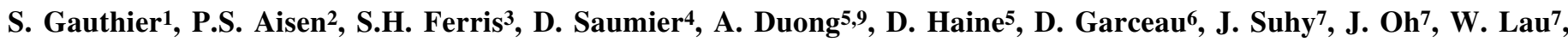 \\ J. Sampalis ${ }^{8}$ for the Alphase Group
}

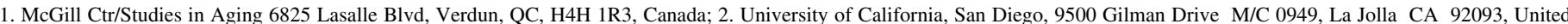

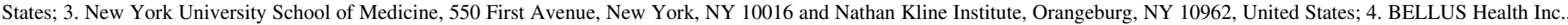

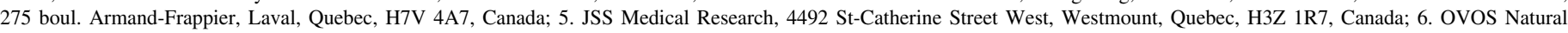

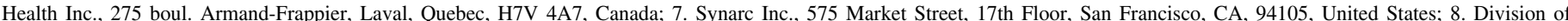

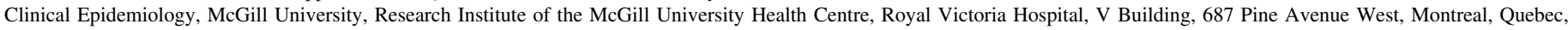
H3A 1A1, Canada. 9. Corresponding author: Anh Duong
\end{abstract}

Erratum for the article "Effect of Tramiprosate in Patients with Mild-to-Moderate Alzheimer's Disease: Exploratory Analyses of the MRI Sub-Group of the Alphase Study" by Gauthier et al., J Nutr Health Aging. 2009 Jul;13(6):550-7. Results section, page 554, second column, the $\mathrm{P}$ values for the change in ADAS-cog scores are given incorrectly. The correct sentence should read as follows (revisions in bold): "Results for the change in ADAS-cog scores showed a marginally significant overall treatment effect $(\mathbf{P}=\mathbf{0 . 0 9 1})$ and a nonsignificant interaction of treatment with time $(\mathbf{P}=\mathbf{0 . 8 8 3})$." These corrections have no impact on the conclusions of the study outcomes. The authors regret the errors. 